\title{
Systemic Therapy for Hepatocellular Carcinoma: Chinese Consensus-Based Interdisciplinary Expert Statements
}

\author{
Yongkun Sun $^{\mathrm{a}}$ Wen Zhang ${ }^{\mathrm{a}}$ Xinyu Bi ${ }^{\mathrm{b}}$ Zhengqiang Yang ${ }^{\mathrm{c}}$ Yu Tang $^{\mathrm{d}}$ Liming Jiang $^{\mathrm{e}}$
} Feng $\mathrm{Bi}^{\mathrm{f}}$ Minshan Chen ${ }^{\mathrm{g}}$ Shuqun Cheng ${ }^{\mathrm{h}}$ Yihebali Chi $^{\mathrm{a}}$ Yue Han ${ }^{\mathrm{c}}$ Jing Huang ${ }^{\mathrm{a}}$ Zhen Huang $^{\mathrm{b}}$ Yuan Ji $\mathrm{J}^{\mathrm{i}}$ Liqun Jia $^{\mathrm{j}}$ Zhichao Jiang ${ }^{\mathrm{a}}$ Jing Jin $^{\mathrm{k}}$ Zhengyu Jin' Xiao $\mathrm{Li}^{\mathrm{c}}$ Zhiyu Li $^{\mathrm{b}}$ Jun Liang ${ }^{\mathrm{m}}$ Lianxin Liu $^{\mathrm{n}}$ Yunpeng Liu ${ }^{\circ}$ Yinying Lu ${ }^{\mathrm{p}}$ Shichun Lu ${ }^{q}$ Qinghua Meng ${ }^{r}$ Zuoxing Niu ${ }^{s}$ Hongming Pan $^{t}$ Shukui Qin ${ }^{u}$ Wang Qu $^{a}$ Guoliang Shao ${ }^{v}$ Feng Shen ${ }^{w}$ Tianqiang Song ${ }^{\mathrm{x}}$ Yan Song $^{\mathrm{a}}$ Kaishan Tao ${ }^{\mathrm{y}}$ Aiping Tian ${ }^{\mathrm{z}}$ Jianhua Wang $^{\mathrm{A}}$ Wenling Wang $^{\mathrm{B}}$ Zhe Wang ${ }^{\mathrm{C}}$ Liqun $\mathrm{Wu}^{\mathrm{D}}$ Feng Xia ${ }^{\mathrm{E}}$ Baocai Xing ${ }^{\mathrm{F}}$ Jianming $\mathrm{Xu}^{\mathrm{G}}$ Huadan Xuel Dong Yan ${ }^{c}$ Lin Yang ${ }^{a}$ Jianming Ying ${ }^{H}$ Jingping Yun' Zhaochong Zeng ${ }^{J}$ Xuewen Zhang $^{\mathrm{K}}$ Yanqiao Zhang ${ }^{\mathrm{L}}$ Yefan Zhang $^{\mathrm{b}}$ Jianjun Zhao ${ }^{\mathrm{b}}$ Jianguo Zhou $^{\mathrm{b}}$ Xu Zhu ${ }^{M}$ Yinghua Zou ${ }^{N}$ Jiahong Dong ${ }^{O}$ Jia Fan ${ }^{\mathrm{P}}$ Wan Yee Lau ${ }^{\mathrm{Q}}$ Yan Sun $^{\mathrm{a}}$ Jinming $\mathrm{Yu}^{\mathrm{R}}$ Hong Zhao $^{\text {b }}$ Aiping Zhou ${ }^{\text {a }}$ Jianqiang Cai ${ }^{b}$

aDepartment of Medical Oncology, National Cancer Center/National Clinical Research Center for Cancer/Cancer Hospital, Chinese Academy of Medical Sciences and Peking Union Medical College, Beijing, China; ${ }^{b}$ Department of Hepatobiliary Surgery, National Cancer Center/National Clinical Research Center for Cancer/Cancer Hospital, Chinese Academy of Medical Sciences and Peking Union Medical College, Beijing, China; 'Department of Interventional Radiology, National Cancer Center/National Clinical Research Center for Cancer/Cancer Hospital, Chinese Academy of Medical Sciences and Peking Union Medical College, Beijing, China; dDepartment of GCP Center, National Cancer Center/National Clinical Research Center for Cancer/Cancer Hospital, Chinese Academy of Medical Sciences and Peking Union Medical College, Beijing, China; eDepartment of Diagnostic Imaging, National Cancer Center/National Clinical Research Center for Cancer/Cancer Hospital, Chinese Academy of Medical Sciences and Peking Union Medical College, Beijing, China; fDepartment of Medical Oncology, West China Hospital, Chengdu, China; ' ${ }^{9}$ epartment of Hepatobiliary Oncology, Sun Yat-sen University Cancer Center, Guangzhou, China; ${ }^{\text {h}}$ The Six Department of Hepatic Surgery, Eastern Hepatobiliary Surgery Hospital, Shanghai, China; 'Department of Pathology, Zhongshan Hospital Fudan University, Shanghai, China; 'Department of Oncology of Integrative Chinese and Western Medicine, China-Japan Friendship Hospital, Beijing, China; ${ }^{k}$ Department of Radiology, National Cancer Center/National Clinical Research Center for Cancer/Cancer Hospital, Chinese Academy of Medical Sciences and Peking Union Medical College, Beijing, China; 'Department of Radiology, Peking Union Medical College Hospital, Beijing, China; 'm Department of Medical Oncology, Peking University International Hospital, Beijing, China; nDepartment of Hepatic Surgery, The First Affiliated Hospital of University of Science and Technology of China, Hefei, China; 'Department of Medical Oncology, The First Affiliated Hospital of China Medical University, Shenyang, China; PDepartment of Comprehensive Liver Cancer Center, The Fifth Medical Center of Chinese PLA General Hospital, Beijing, China; 'Department of Hepatobiliary Surgery, First Medical Center of Chinese People's Liberation Army (PLA) General Hospital, Chinese PLA Medical School, Beijing, China; 'Department of Clinical Care Medicine

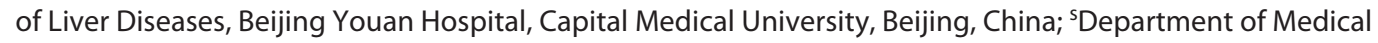

Yongkun Sun, Wen Zhang, Xinyu Bi, Zhengqiang Yang, Yu Tang, and Liming Jiang contributed equally to this work.

Karger@karger.com www.karger.com/lic Karger ${ }^{\prime \prime}=$ BOPEN ACCESS
(C) 2022 The Author(s)

Published by S. Karger AG, Basel

This is an Open Access article licensed under the Creative Commons Attribution-NonCommercial-4.0 International License (CC BY-NC) (http://www.karger.com/Services/OpenAccessLicense), applicable to the online version of the article only. Usage and distribution for commercial purposes requires written permission.
Correspondence to:

Hong Zhao, zhaohong@ cicams.ac.cn

Aiping Zhou, aiping_zhou@ @eah.net

Jianqiang Cai , caijianqiang188@sina.com 


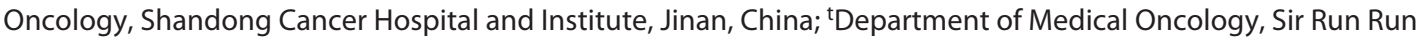
Shaw Hospital, College of Medicine, Zhejiang University, Hangzhou, China; "Department of Medical Oncology, PLA Cancer Centre of Nanjing Bayi Hospital, Nanjing, China; 'Department of Interventional Radiology, Cancer Hospital of the University of Chinese Academy of Sciences, Hangzhou, China; wDepartment of Hepatic Surgery IV, Eastern Hepatobiliary Surgery Hospital, Shanghai, China; ${ }^{x}$ Department of Hepatobiliary Surgery, Tianjin Medical University Cancer Institute and Hospital, Tianjin, China; ' ${ }^{\vee}$ Department of Hepatobiliary Surgery, Xijing Hospital, Fourth Military Medical University, Xi'an, China; ${ }^{2}$ Department of Traditional Chinese Medicine, National Cancer Center/National Clinical Research Center for Cancer/Cancer Hospital, Chinese Academy of Medical Sciences and Peking Union Medical College, Beijing, China; A Department of Interventional Radiology, Zhongshan Hospital Fudan University, Shanghai, China; ${ }^{B}$ Department of Radiology, The Affiliated Hospital of Guizhou Medical University, Guizhou Cancer Hospital, Guiyang, China; 'Department of Orthopedics, Xijing Hospital, Fourth Military Medical University, Xi'an, China; DDepartment of Hepatic Biliary Pancreatic Surgery, The Affiliated Hospital of Qingdao University, Qingdao, China; EDepartment of Hepatobiliary Surgery, The Southwest Hospital of AMU, Chongqing, China; FDepartment of Hepatobiliary and Pancreatic Surgery Unit I, Beijing Cancer Hospital, Beijing, China; ${ }^{\top}$ Department of Gastrointestinal

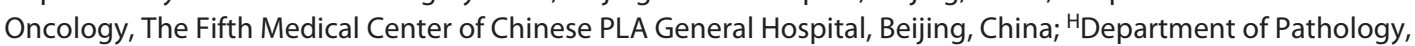
National Cancer Center/National Clinical Research Center for Cancer/Cancer Hospital, Chinese Academy of Medical Sciences and Peking Union Medical College, Beijing, China; 'Department of Pathology, Sun Yat-sen University Cancer Center, Guangzhou, China; 'Department of Radiology, Zhongshan Hospital Fudan University, Shanghai, China; ${ }^{K}$ Department of Hepatobiliary and Pancreatic Surgery, Second Hospital of Jilin University, Changchun, China; ${ }^{L}$ Department of Oncology, Harbin Medical University Cancer Hospital, Harbin, China; ${ }^{M}$ Department of Interventional Radiology, Beijing Cancer Hospital, Beijing, China; NDepartment of Interventional Radiology, Peking University First Hospital, Beijing, China; ${ }^{\circ}$ Department of Hepatopancreatobiliary Surgery, Beijing Tsinghua Changgung Hospital, Beijing, China; PDepartment of Liver Surgery, Zhongshan Hospital Fudan University, Shanghai, China; ${ }^{Q}$ Department of Hepatic Biliary Pancreatic Surgery, The Chinese University of Hong Kong, Hong Kong, China; ${ }^{\text {RDepartment of }}$ Radiology, Shandong Cancer Hospital and Institute, Shandong First Medical University and Shandong Academy of Medical Science, Taian, China

\section{Keywords}

Chinese interdisciplinary experts $\cdot$ Hepatocellular carcinoma $\cdot$ Molecular targeted therapy $\cdot$ Consensus

\begin{abstract}
Background: Hepatocellular carcinoma (HCC) is the most common type of liver cancer and causes many cancer-related deaths worldwide; in China, it is the second most prevalent cause of cancer deaths. Most patients are diagnosed clinically with advanced stage disease. Summary: For more than a decade, sorafenib, a small-molecular-weight tyrosine kinase inhibitor (SMW-TKI) was the only molecular targeted drug available with a survival benefit for the treatment of advanced HCC. With the development of novel TKIs and immune checkpoint inhibitors for advanced HCC, the management of patients has been greatly improved. However, though angiogenic-based targeted therapy remains the backbone for the systemic treatment of HCC, to date, no Chinese guidelines for novel molecular targeted therapies to treat advanced HCC have been established. Our interdisciplinary panel on the treatment of advanced HCC comprising
\end{abstract}

Chinese Expert Statements on Molecular

Targeted Therapy for HCC hepatologists, hepatobiliary surgeons, oncologists, radiologists, pathologists, orthopedic surgeons, traditional Chinese medicine physicians, and interventional radiologists has reviewed the literature in order to develop updated treatment regimens. Key Messages: Panel consensus statements for the appropriate use of new molecular -targeted drugs including doses, combination therapies, adverse reaction management as well as efficacy evaluation, and predictions for treatment of advanced HCC with evidence levels based on published data are presented, thereby providing an overview of molecular targeted therapies for healthcare professionals.

(C) 2022 The Author(s).

Published by S. Karger AG, Basel

\section{Introduction}

Globally, hepatocellular carcinoma (HCC) is one of the main causes of cancer-related mortality. In 2015, morbidity and mortality in China were 370,000 and 326,000 people, respectively [1]. HCC is clinically characterized by an unperceived onset, rapid progression, early 


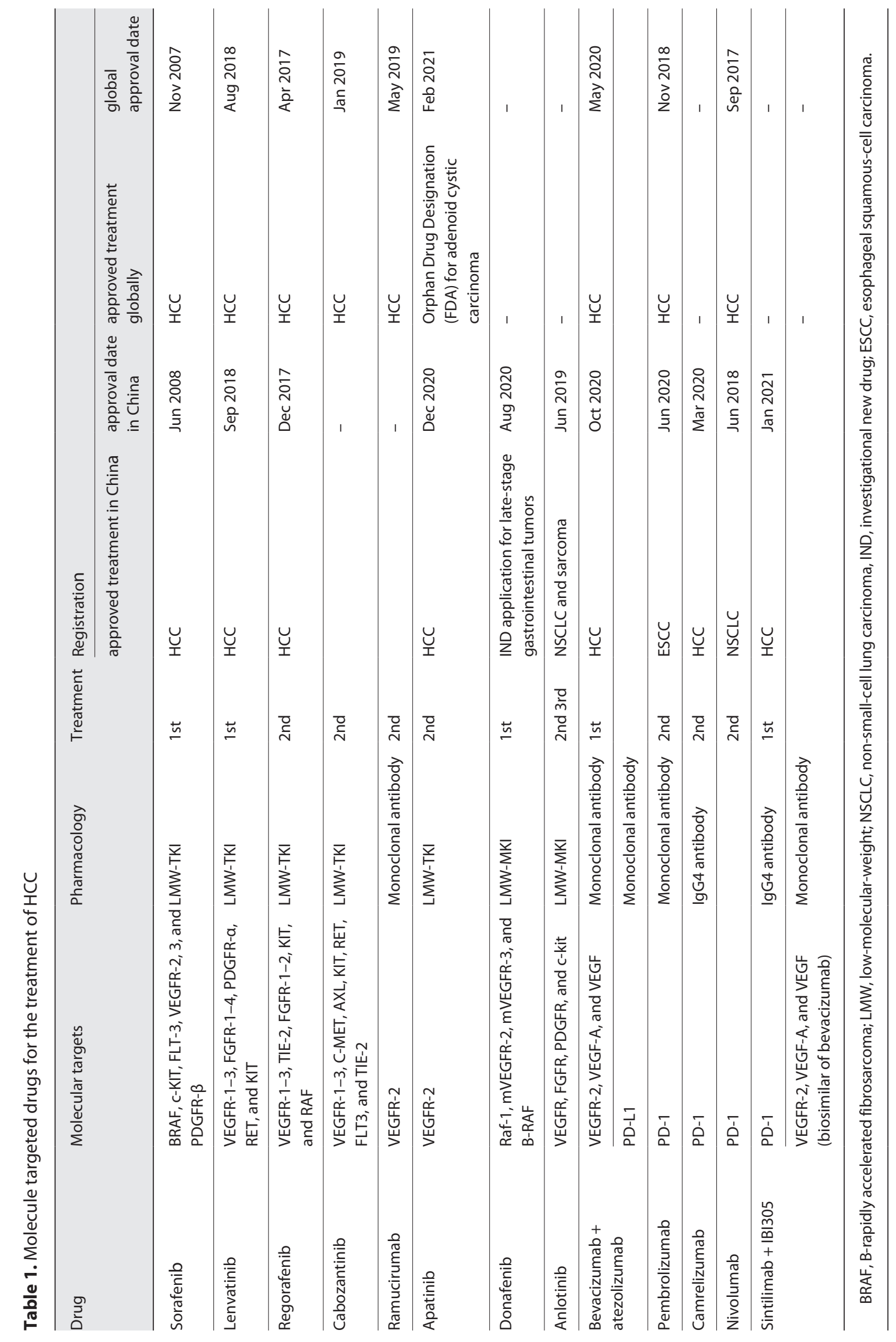


Table 2. Levels of evidence for therapeutic studies

\begin{tabular}{ll}
\hline $\begin{array}{l}\text { Level of } \\
\text { evidence }\end{array}$ & \begin{tabular}{l} 
Investigating the results of treatment (therapeutic studies) \\
\hline 1
\end{tabular} \\
$\begin{array}{l}\text { High-quality randomized trial with a statistically significant difference or no statistically significant difference but narrow } \\
\text { confidence intervals; systematic review of level 1 RCTs (the study results were homogenous) }\end{array}$ \\
\hline $\begin{array}{l}\text { Lesser quality RCT (e.g., <80\% follow-up, no blinding, or improper randomization), prospective comparative study, } \\
\text { systematic review of level } 2 \text { or level } 1 \text { studies with inconsistent results }\end{array}$ \\
\hline \begin{tabular}{l} 
Case control study, retrospective comparative study, and systematic review of level 3 studies \\
\hline 5
\end{tabular} & Case series \\
\hline
\end{tabular}

recurrence, and late-stage diagnosis and is difficult to treat, resulting in a poor prognosis.

In recent years, significant advances have been made in the development of molecular targeted drugs for HCC therapy [2]. In 2007, the FDA in the USA approved tyrosine kinase inhibitors (TKIs) sorafenib for the therapy of advanced HCC. Subsequently, worldwide approval was given for the use of several other oral multi-targeted TKIs to treat HCC. In China, the development of molecular targeted therapies for advanced HCC began in 2006. Data from the Asia-Pacific ORIENTAL trial that compared sorafenib versus placebo were given in 2008 at the American Society of Clinical Oncology, which finally lead to its approval in the same year. Thereafter, more than 160,000 patients have been treated in China with sorafenib being the gold standard for those diagnosed with advanced stage disease.

The new multi-targeted TKIs, regorafenib, lenvatinib, and donafenib, which became available in China after 2017, have expanded the options for the treatment of advanced HCC and provided effective therapy for recurrent/progressive disease. Additionally, apatinib is a novel molecular targeted drug for HCC treatment and has been provided by Chinese companies in recent years [3]. Anlotinib has been proven to be effective also for HCC, though it is still under investigation in phase 2 clinical trials. Also, ramucirumab and cabozantinib are, in China, still under investigation after they were approved by the FDA for HCC treatments 2 years before, but they can serve as indicators for HCC treatments in China within the next years. Other molecular targeted therapies include programmed cell death protein (PD-1) inhibitors as mono- or combination therapies (Table 1). In the era of immunotherapy, molecular targeted therapy and immunotherapy are both novel therapies for HCC and mo- lecular targeted therapy combined with immunotherapy as systemic treatment has become a new approach for advanced HCC. However, the CKECKMATE-459 [4] and KEYNOTE240 [5] studies revealed that molecular targeted therapy is still the major cornerstone treatment for advanced HCC. We took targeted therapy as the entry point to guide the application selection of targeted therapy drugs in different scenarios, while a consensus on the application of immunotherapy will be introduced in the future.

How to select the currently available molecular targeted drugs under various disease status and how to combine them with other drugs is one of the remaining critical issues in treating advanced HCC. The interdisciplinary expert panel consensus statements are based on the clinical symptoms of HCC in China, clinical trial outcomes, experience with current molecular targeted therapies regarding adverse events (AEs), drug dose selection, efficacy evaluation, efficacy predictions, and multidisciplinary collaboration treatment experiences. The aim of our expert panel statements was to guide physicians, patient's advocates, healthcare providers, and medical institutions in the appropriate application of molecular targeted therapies for advanced HCC.

\section{Methods}

The levels of clinical evidence and the strength of statements given by the expert panel are applied strictly to Chinese practice. Consensus was oriented based on the European Society for Medical Oncology guiding principles for HCC published in 2018 [6] and the Guidelines for the Diagnosis and Treatment of Hepatocellular Carcinoma of the Chinese Society of Clinical Oncology published in 2020. Five levels of evidence were graded according to the therapeutic studies and four degrees of statement implemented 
Table 3. Definition of recommendation

\begin{tabular}{ll}
\hline Recommendation grade & Description \\
\hline 1 & $\begin{array}{l}\text { Good evidence (level } 1 \text { studies with consistent findings) for or against recommending intervention; treatment } \\
\text { measures with good accessibility in China }\end{array}$ \\
\hline $\begin{array}{l}\text { Fair evidence (level } 2 \text { or } 3 \text { studies with consistent findings) for or against recommending intervention but with } \\
\text { limited clinical benefit or poor accessibility in China }\end{array}$ \\
\hline $\begin{array}{l}\text { Conflicting or poor-quality evidence (level } 4 \text { or } 5 \text { studies) not allowing a recommendation for or against } \\
\text { intervention with poor accessibility in China }\end{array}$ \\
\hline (no recommendation) & There was insufficient evidence to make a recommendation \\
\hline
\end{tabular}

and classified (Tables 2, 3). Molecular targeted therapy is defined as a drug which interferes with specific targeted molecules and is differentiated from hormonal or chemotherapies.

\section{Therapies for Advanced HCC}

\section{Single Molecular Targeted Monotherapies}

First-Line TKI Medications

The SHARP research [7] and the Asia-Pacific study [8] proved sorafenib to have good efficacy and well tolerated as first-line therapy for patients with advanced stage HCC disease. The SHARP study enrolled 602 patients. The median overall survival (OS) time for sorafenib was 10.7 months compared to 7.9 months for the placebo $(\mathrm{HR}=$ 0.69). The median time to progression (TTP) was 2.8 months for the placebo and 5.5 months for sorafenib. The ORIENTAL study enrolled 271 patients randomized at a 2:1 ratio to be given sorafenib or a placebo. The median OS time was 6.5 and 4.2 months $(\mathrm{HR}=0.68)$, and the median TTP 2.8 and 1.4 months, respectively. Despite differences between ethnicities of the enrolled populations in the two studies, sorafenib produced basically the same OS time benefit.

The REFLECT study enrolled 954 medically untreated patients with advanced HCC who were randomly allocated to either lenvatinib or sorafenib therapy. The median OS times in the two groups were 13.6 and 12.3 months, respectively, with the data showing that lenvatinib was not inferior to sorafenib (HR $=0.92,95 \% \mathrm{CI}$ : $0.79-1.06$ ), a finding consistent with the primary endpoint. Progression-free survival (PFS) in the two groups was 7.4 and 3.7 months, respectively $(p<0.000$, HR = $0.66,95 \%$ CI: $0.57-0.77)$. Analyzing separately the subgroups for lenvatinib and sorafenib for mainland China, Taiwan, and Hong Kong, 144 patients were involved. The
OS times were 15.0 and 10.2 months, and the PFS times were 9.2 and 3.6 months, respectively. In this study, the rates of treatment-emergent $\mathrm{AEs} \geq 3$ were similar in both groups (episodes per patient-year 3.2 in the lenvatinib group vs. 3.3 in the sorafenib group). The most common treatment-related AEs (TRAEs) were slightly different. Hypertension and decreased appetite occurred more frequently in those patients treated with lenvatinib, yet palmar-plantar erythrodysesthesia was more common in the sorafenib group [9].

The ZGDH3 study [10] involved 668 randomized patients who received donafenib or sorafenib. The main endpoint was OS, and the median OS in the two groups was 12.1 and 10.3 months, respectively $(p=0.036)$. Secondary endpoints were PFS and response rates, which were 3.7 and 3.6 months $(p=0.282)$, and response rates $4.6 \%$ and $2.7 \%(p=0.245)$, respectively. The rate of TRAEs of grade $\geq 3$ was $57.4 \%$ and $67.5 \%(p=0.008)$, respectively. Compared with sorafenib, donafenib produced prolonged survival and caused fewer TRAEs.

Another first-line phase 2 study of advanced HCC enrolled 121 patients to receive apatinib $750 \mathrm{mg}$ qd or 850 mg qd [11]. The median TTP was 3.32 and 4.21 months, and the median OS 9.82 and 9.71 months, respectively. No difference was detected between doses $(p>0.05)$, and apatinib at $750 \mathrm{mg}$ po qd was recommended for phase 3 studies.

\section{Future Perspective}

A phase 2 study using anlotinib for advanced HCC included 50 patients, with 2 cohorts receiving first- and second-line treatments. For the first-line treatment, the 12week PFS rate was $80.8 \%$, with a median TTP of 5.9 months and an OS time of 12.8 months [12].

Consensus-based statement 1: For first-line treatment of locally advanced Barcelona Clinic Liver Cancer (BCLC) 
stages $B$ or $C$ not suitable for local treatment, the options are sorafenib (grade 1 recommendation; evidence level 1) lenvatinib (grade 1 recommendation, evidence level 1) and donafeniib (grade 1 recommendation, evidence level 1); apatinib (grade 3 recommendation, evidence level 2).

Ongoing molecular targeted therapy options: Anlotinib (grade 3 recommendation, evidence level 3).

\section{Second-Line TKI Medication}

Regorafenib, apatinib, cabozantinib, and ramucirumab were investigated as second-line treatments in phase 3 trials. The RESORCE study included 573 HCC patients with Child-Pugh score A, who failed to respond to but could tolerate sorafenib ( $\geq 400 \mathrm{mg}$ daily for at least 20 of the 28 days before discontinuation). Patients were randomized (2:1 ratio) to be given either regorafenib or best supportive care [13]. The median OS times for regorafenib and best supportive care were 10.6 and 7.8 months $(\mathrm{HR}=0.63)$, median PFS times 3.1 and 1.5 months, and response rates $10.6 \%$ and $4.1 \%$, respectively.

The AHELP phase 3 study using apatinib as the second-line treatment [14] was conducted in 31 centers in China. A total of 393 patients who received at least one line of systemic therapy (including sorafenib- and oxaliplatin-based chemotherapy), were enrolled from April 2014 to May 2017 and randomized at a ratio of 2:1 to receive apatinib at $750 \mathrm{mg}$ or a placebo. The median OS times in the two groups were 8.7 and 6.8 months ( $p=$ $0.048)$, median PFS 4.5 and 1.9 months $(p<0.000)$, and response rates $10.7 \%$ and $1.5 \%$, respectively. The most frequently occurring grades 3 to 4 TRAEs for apatinib were hypertension (27.6\%), hand-foot-skin reactions (17.9\%), thrombocytopenia (13.2\%), and neutropenia (10.5\%). Apatinib significantly improved OS and PFS but elicited a greater number of AEs.

The CELESTIAL study enrolled 707 patients with up to two previous systemic and sorafenib HCC treatments, and after randomization (ratio 2:1), they received either cabozantinib or a placebo. The median OS times were 10.2 and 8 months, and median PFS 5.2 and 1.9 months, respectively [15]. The REACH-2 study enrolled 292 patients, with an alpha fetal protein (AFP) level $\geq 400 \mu \mathrm{g} / \mathrm{L}$, who failed to respond to sorafenib and were randomized (ratio 2:1) to be given either ramucirumab or a placebo [16]. The median OS times for ramucirumab or placebo were 8.5 and 7.3 months, and median PFS 2.8 and 1.6 months, respectively. The benefits of ramucirumab in the REACH-2 study were more evident in patients with AFP concentrations $\geq 400 \mu \mathrm{g} / \mathrm{L}$, pointing to AFP as being potentially predictive. Anlotinib as second-line treatment in

Chinese Expert Statements on Molecular

Targeted Therapy for HCC a phase 2 study lead to a 12 -week PFS rate of $72.5 \%$ and a median TTP of 4.6 months, while the median OS time was 18 months [12].

Consensus-based statement 2: After failed first-line sorafenib treatment of locally advanced BCLC stage $B$ and advanced BCLC stage C not suitable for local treatment, options are regorafenib (grade 1 recommendation, evidence level 1); apatinib (grade 2 recommendation, evidence level 1); There has been no established second-line treatment for lenvatinib. Effective post treatments after lenvatinib should be established from clinical practice; ongoing molecular targeted therapy options cabozantinib (grade 2 recommendation, evidence level 1), ramucirumab (grade 2 recommendation, evidence level 1); and anlotinib (grade 3 recommendation, evidence level 3 ).

\section{Systemic Combination Therapies}

Systemic Combination Therapies including

Molecular Targeted and Immune Checkpoint

Inhibitor Medications

In recent years, immune checkpoint inhibitors (ICIs) such as PD-1 inhibitors have shown remarkable efficacy in the treatment of lung cancer and others such as malignant melanoma and lymphoma. Starting in 2017, clinical studies using ICIs to treat advanced HCC were conducted and lead to a breakthrough in second-line therapies. Atezolizumab combined with bevacizumab versus sorafenib investigated in the phase 3 IMbrave150 study showed a significant prolongation of OS and PFS. The median OS times were 19.2 months with atezolizumab-bevacizumab versus 13.4 months with sorafenib, while median PFS times were 6.8 months and 4.3 months, respectively. Grade 3 or 4 treatment-related AEs occurred in $43 \%$ of patients treated with atezolizumab-bevacizumab and in $46 \%$ of patients treated with sorafenib [17] Based on the outcome of IMbrave150, atezolizumab combined with bevacizumab has become a new option for the first-line treatment of Child-Pugh grade A advanced HCC.

Orient-32 were randomized, open-label, multicenter phase 2 and 3 clinical trials that investigated the safety and efficacy of sintilimab combined with bevacizumab biosimilar drugs as first-line treatments for Chinese patients with advanced HCC compared to sorafenib alone. The latter study randomized patients in a 2:1 ratio with 380 patients given sintilimab combined with the bevacizumab biosimilar and 191 patients given sorafenib. The median follow-up time was 10.0 months. Compared with sorafenib, the median OS was significantly prolonged in the sintilimab combined with the bevacizumab biosimilar group (not estimable vs. 10.4 months, HR 0.57, 95\% CI: 
$0.43-0.75, p<0.001$ ), and PFS (4.6 vs. 2.8 months, HR $0.56,95 \%$ CI: $0.46-0.70, p<0.001)$ was significantly improved. The incidence rate of TRAEs grade $3 / 4$ was $33.7 \%$ for the combination treatment group and $35.7 \%$ for the sorafenib group, respectively. Taken together, compared with sorafenib, first-line treatment of sintilimab combined with a bevacizumab biosimilar provided significant clinical benefits for Chinese patients [18].

In addition, a number of phase $1 / 1 \mathrm{~b}$ studies on other molecular targeted drug combinations with ICIs have been conducted. Keynote 524 explored pembrolizumab combined with lenvatinib as first-line treatment of 100 patients. Preliminary results presented at the American Society of Clinical Oncology (2020) reported an objective response rate (ORR) of 36\% (95\% CI: 26.6-46.2), median OS 22.0 months (95\% CI: 20.4 to NE), and median PFS 8.6 months (95\% CI: 7.1-9.7) [19]. The incidence of grade $\geq 3$ TRAEs was $67 \%$ with grades $\geq 4$ only $4 \%$. The most common grade 3 TRAE was hypertension (17\%).

A phase 2 study (RESCUE) of camrelizumab combined with apatinib for advanced HCC was conducted at 25 centers in China, and results were reported at the ESMO 2020 conference, about patients with advanced HCC who had not been given or therapy had failed with sorafenib or donafenib. Enrolled patients received intravenous camrelizumab $200 \mathrm{mg}$ and apatinib $250 \mathrm{mg} \mathrm{qd}$ every 2 weeks. The study included 70 first-line and 120 second-line treatment patients, and 168 of 190 patients (88\%) had hepatitis B virus (HBV) infections. According to Response Evaluation Criteria for Solid Tumors (RECIST) ver. 1.1, the ORRs evaluated by an independent review board were $34 \%$ and $23 \%$; according to modified RECIST (mRECIST) independent assessments, ORRs were $46 \%$ and $25 \%$, respectively. The OS rates of 12 months were $75 \%$ and $68 \%$, respectively. The updated results were reported at the ASCO 2021 conference; the median OS times were 20.1 months in first-line and 21.8 months in second-line treatments [20]. Overall, 147 (77\%) patients had TRAE grade $\geq 3$, most commonly hypertension (34\%) and elevated $\gamma$-GT (12\%). Taken together, combination treatment attempts achieved high response rates in both first-line and second-line treatments for HCC, and the combination of camrelizumab and apatinib may be a promising strategy for the therapy of patients with advanced HCC; further trials should confirm the preliminary data.

In addition, studies on pembrolizumab combined with regorafenib and nivolumab combined with lenvatinib have reported ORRs of $26-54.2 \%$, but reliable survival data have not been published yet [21-23]. Updated results were reported in the 2021 ASCO annual meeting, which presented a phase $1 \mathrm{~b}$ study of regorafenib $120 \mathrm{mg} /$ day plus pembrolizumab for first-line treatment of advanced HCC with a median OS time of 26.5 months, a median PFS time of 7.5 months, and a median TTP of 8.1 months [24]. Currently, a number of phase 3 studies that are comparing pembrolizumab combined with lenvatinib versus lenvatinib, camrelizumab combined with apatinib versus sorafenib are ongoing.

As prerequisites for the application of combination therapies of molecular targeted drugs and ICIs, patients should be strictly screened for liver function and bleeding risks and concomitant medical disorders. For patients with confirmed $\mathrm{HBV}$ or hepatitis $\mathrm{C}$ virus ( $\mathrm{HCV}$ ) infections, antiviral therapy should be used to control HBV DNA and HCV RNA titers before checkpoint inhibitor therapy, and active viral hepatitis should be closely monitored during subsequent immunotherapy. Defining the patients risk for TRAEs is critical since combination therapies are more toxic than single-agent therapies and dose adaption may be clinically appropriate. For example, in the study of camrelizumab combined with apatinib, the recommended dose of apatinib was only $250 \mathrm{mg}$.

Consensus-based statement 3: For first-line treatment of locally advanced BCLC stage B and advanced BCLC stage $C$ patients with Child-Pugh grade A not suitable for local treatment options, the preferred treatment is antiangiogenesis drugs as the main medication combined with immunocheckpoint inhibitors including bevacizumab combined with atezolizumab, or sintilimab combined with the bevacizumab biosimilar (grade 1 recommendation, evidence level 1).

Systemic Therapies including Molecular Targets

Combined with Other Drugs

A randomized phase 3 clinical study (SEARCH) has shown that sorafenib combined with erlotinib did not improve OS compared to sorafenib/placebo for patients with advanced HCC [25]. The CALGB 80802, another phase 2 study, compared sorafenib combined with doxorubicin versus doxorubicin alone [26]. Median OS times for the combination and monotherapy were 8.9 and 10.5 months, respectively but without a statistically significant difference. A phase 2 study on sorafenib combined with other chemotherapeutics such as gemcitabine [27] and 5-fluorouracil [28] found ORRs of 3-4\%, median OS times of 11.6-13.7 months and TTPs of 3.6-8.0 months. When sorafenib was administered with gemcitabine and oxaliplatin, the median OS was 15.7 and for TTP 10.3 months, respectively [29]. These 
promising results however should be confirmed by larger clinical studies.

Consensus-based statement 4: There is currently no evidence that the combination of different molecular targeted drugs or a combination of molecular targeted drugs and chemotherapy can significantly improve survival in advanced HCC (not recommended, evidence level 1).

\section{Molecular Targeted Therapy as Adjuvant Treatment for HCC}

Since sorafenib has become the gold standard firstline therapy for patients with advanced HCC, it is reasonable to investigate molecular targeted agents postoperatively in the adjuvant setting [30]. The STORM study, which was a randomized phase 3 study, enrolled 1,114 patients after radical resection. Patients were randomly given either sorafenib $(n=556)$ or a placebo $(n$ $=558$ ). There were no statistically significant differences in median OS or PFS times between the two groups, and therefore, no improved survival or reduced recurrence rates resulted, probably because of the inclusion of many patients at a low risk of HCC recurrence. In a study from Taiwan that focused on patients at a high risk of recurrence (poor differentiation, microvascular invasion, and satellite nodules), patients were randomized postoperatively to receive either sorafenib $400 \mathrm{mg}$ qd for 4 months or necessary antiviral therapy and supportive care. Here, the rate of recurrence was significantly less in the sorafenib group than in the controls $(29.4 \%$ vs. $70.7 \%, p=0.032)$. Multivariate analysis suggested that sorafenib was an independent risk factor for postoperative recurrence [31]. A retrospective study of 728 patients conducted by the team of Professor Cheng Shuqun at Eastern Hepatobiliary Hospital revealed that adjuvant sorafenib following radical resection improved OS and PFS times for high-risk patients with microvascular invasion [32]. Other studies performed in China also indicated that adjuvant sorafenib improved OS times in patients with intermediate/advanced BCLC stages B or C, the drug being well tolerated by patients. These studies however may be criticized due to the small sample sizes, the retrospective setting, and the low level of evidence [33-35]. A recent multicenter prospective cohort study also revealed that combined use of lenvatinib and transarterial chemoembolization (TACE) can prolong the disease-free survival of HCC patients with high recurrence risk after resection [36]. However, biases resulting from the nonrandomized design and small sample size decreased the reliability of this evidence. Therefore, well-designed, large-sample, prospective, randomized multicenter clinical studies are still needed for validation.

Consensus-based statement 5: For patients with lowrisk of recurrence BCLC stage 0, stage A, adjuvant molecular targeted therapies are not recommended after radical resection (not recommended, evidence level 1).

Consensus-based statement 6: For patients with a high risk of recurrence of $B C L C$ stage $B$ or $C$, or with high-risk factors such as microvascular invasion, poor differentiation, satellite nodules, sorafenib or lenvatinib might play some role after radical resection (grade 3 recommendation, evidence level 3).

\section{Molecular Targeted Therapies after Liver}

\section{Transplantation}

Clinical studies have shown that molecular targeted drugs such as sorafenib for systemic treatment or combined with TACE, radiofrequency ablation (RFA), and other local measures can be considered for patients with unresectable HCC relapsing after liver transplantation [37-41]. In the case of first-line sorafenib therapy failure, switching to second-line regorafenib can prolong the survival time [42]. Lenvatinib also seems be effective in patients relapsing after liver transplantation, but confirmative investigations are needed.

After liver transplantation, commonly used immune suppressants are calcineurin inhibitors such as cyclosporine and tacrolimus. It is noteworthy that these drugs are independent risk factors for the recurrence of HCC after a liver transplant $[43,44]$. The recurrence rate of HCC in liver transplant patients on mammalian target of rapamycin inhibitors is significantly lower than the rate in patients on calcineurin inhibitors. The recurrence rate of HCC is even lower in liver transplant recipients treated with sirolimus. Sirolimus-based immunosuppressive regimens combined with sorafenib are therefore recommended for patients with recurrent HCC who have received a liver transplant $[45,46]$. ICIs are not recommended because of their immune stimulating effects, their negative effects on immune suppression, and their graft rejection potential.

Consensus-based statement 7: For patients with unresectable HCC who relapsed after liver transplantation, the options are to use molecular targeted drugs such as sorafenib and regorafenib combined with TACE, RFA and other advanced techniques; a sirolimus-based regimen is recommended as a preferred immunosuppressive treatment (grade 1 recommendation, evidence level 2).

Postoperative adjuvant systemic therapies included sorafenib, as well as doxorubicin, gemcitabine, cisplatin, 
and other chemotherapeutics, for tumors with high-risk factors for recurrence, such as a tumor stage exceeding the Milan indication criteria, poorly differentiated histology, blood vessel invasion, and lymph node metastases. Nonrandomized studies of adjuvant therapies for high-risk patients after liver transplantation showed that sorafenib was more effective than oral gemcitabine. Therefore, sorafenib should be considered as an adjuvant treatment in patients with high-risk factors who are undergoing liver transplantation [47-49]. Metuximab (licartin) may also be considered as an adjuvant therapy, but confirmative data of prospective studies are still lacking.

Consensus-based statement 8: For patients undergoing liver transplantation who have any of the following risk factors: exceeded the Milan indication criteria; poorly differentiated histology, blood vessel invasion; and lymph node metastases, adjuvant molecular targeted therapy might be an option to decrease the risk of recurrence (grade 3 recommendation, evidence level 4).

\section{Local Ablation Combined with Molecular Targeted Therapies}

RFA is an appropriate treatment to inhibit early-stage HCC, with the advantage of the therapy being less traumatic and deteriorative to liver functions, but being definitively curative and producing reproducible outcomes. Patients with China liver cancer stage Ia HCC and some patients with stage Ib (i.e., single tumor diameter $\leq 5.0 \mathrm{~cm}$ or 2-3 tumors with a maximum diameter of $\leq 3.0 \mathrm{~cm}$ ), as well as patients with no vascular invasion, no bile duct, adjacent organ invasion, distant metastasis, and a liver function Child-Pugh grade A or B are candidates for RFA [50]. The aim of an upcoming clinical trial, which will add molecular targeted therapies to RFA, is the prevention of disease recurrence. However, based on current evidence, the use of molecular targeted drugs for adjuvant treatment after RFA cannot be recommended. In the STORM study 1,114 patients from 202 centers were randomized in a ratio of $1: 1$ to postoperative adjuvant sorafenib or a placebo following surgical resection $(n=900)$ and RFA ( $n$ $=214$ ). No difference was found in the median PFS between the two groups (33.3 and 33.7 months, respectively) [30]. The role of newer molecular targeted agents such as lenvatinib and regorafenib has to date not yet been defined clinically. Palliative ablation therapy for advanced HCC is currently under investigation. Nevertheless, combining TACE ablation and sorafenib has shown longer survival for patients with BCLC stage C [51].
Consensus-based statement 9: For patients with earlystage HCC; adjuvant molecular targeted therapies after local ablation is not recommended (not recommended, evidence level 2).

\section{External Beam Radiation Combined with Molecular}

\section{Targeted Therapy}

Clinical trials on radiotherapy combined with molecular targeted therapy mainly included patients with intermediate and advanced stage disease. A phase 2 study enrolled 40 patients with locally advanced HCC that was unresectable and unsuitable for TACE. Patients received conventional radiotherapy and sorafenib during and after radiation until disease progression [52]. The ORR of the entire group of patients was $55 \%$ at 1 month after radiotherapy and 2 -year OS rates were $32 \%$ and $39 \%$, respectively. The incidence of $\geq 2$ grade hepatotoxicity was $35 \%$, including 3 deaths, indicating a potential risk of the concomitant treatment strategies. A retrospective analysis found that the incidence of grade 3 TRAEs was high [53]. The RTOG1112 phase 3 trial which compared sorafenib versus sorafenib to treat patients with stereotactic body radiotherapy (SBRT), who had HCC that was locally advanced, is ongoing and the outcome is eagerly awaited.

A South Korean phase 2 study investigated sequential setting enrolled 47 patients with BCLC stage C [54]. Patients received conventionally fractionated radiotherapy for intrahepatic lesions. Continuous 5-FU and tetrahydrofolate hepatic artery pump chemotherapy were given for 5 days before and after radiotherapy, and oral sorafenib was started 4 weeks after radiotherapy. The median OS time of the entire group was 24.6 months (95\% CI: 10.938.4), and the AEs were manageable. A Japanese retrospective analysis also reported that intrahepatic radiotherapy combined with hepatic artery pump chemotherapy followed by sequential sorafenib improved survival [55].

Consensus-based statement 10: For patients with inoperable BCLC stages $B$ or $C$ without extrahepatic metastases, concurrent radiotherapy and sorafenib should be used cautiously due to toxicity. Sequential sorafenib following radiation can be considered, but confirmative large clinical studies are still missing (grade 3 recommendation, evidence level 3).

A prospective study compared sorafenib combined with intrapulmonary SBRT versus SBRT alone in 60 patients with lung metastases of HCC [56]. Median PFS and OS times were improved by the combination therapy with acceptable toxicity, suggesting that SBRT could be 
safely and effectively used for oligo-metastatic disease together with targeted therapies. A retrospective analysis from Fudan University Zhongshan Hospital also found that patients with lung metastases of HCC who received radiotherapy (helical tomotherapy) combined with sorafenib had a significantly better outcome compared to those patients who received only radiotherapy (median OS time: 29.6 vs. 23 months, $p=0.031$ ) or targeted therapy alone (median OS: 29.6 months vs. 25 months, $p=$ 0.018 ) [57].

Consensus-based statement 11: For patients receiving molecular targeted therapy for extrahepatic metastases the addition of SBRT is an option in the case of oligo-metastatic disease (grade 3 recommendation, evidence level 3).

\section{TACE Combined with Molecular Targeted Therapy}

TACE alone is appropriate therapy for some patients that exhibit early-stage or mid-stage HCC (BCLC stages 0 , A or B; or stages I-II in the Chinese Guidelines for the Diagnosis and Treatment of this disease [2020]), who are not suitable for surgical resection because of reduced liver functions. Adding sorafenib to drug-eluting bead TACE (DEB-TACE) failed to prolong the TTP [58].

Adding sorafenib to DEB-TACE also failed to improve PFS [59] in the TACE2 trial, a phase 3 study that included 399 patients with BCLC stage B from 20 UK hospitals. Median PFS for DEB-TACE plus sorafenib was 238 days (95\% CI: 221.0-281.0 days) and for DEB-TACE plus placebo 235 days (95\% CI: 209.0-322.0 days), respectively, resulting in a HR of 0.99 (95\% CI: $0.77-1.27, p=0.940$ ). In contrast, a Japanese TACTICS phase 2 study that used an uncommon design indicated that TACE plus sorafenib could improve PFS in patients with early-stage and midstage unresectable HCC but without vascular invasion and extrahepatic metastases, suggesting grade 3 level recommendation [60]. In this study, 197 patients with unresectable HCC but no extrahepatic metastases were enrolled in 33 hospitals. The diameter of tumors was $\leq 10$ $\mathrm{cm}$, and the number of lesions should have been not more than 10, without vascular invasion. Thirty eight percent of the patients had BCLC stage A and 50\% stage B, respectively. The main study endpoint was PFS defined by the time to untreatable (unTACEable) progression. This unique study endpoint has the potential of delaying the time to change treatment strategies, reflecting todays TACE real-world practice, meaning that if new lesions appear in the liver during TACE treatment, TACE would be continued and thus prolong the time of effective therapy. The median PFS time reached 25.2 months by adding sorafenib to conventional TACE versus 13.5 months

Chinese Expert Statements on Molecular

Targeted Therapy for HCC for conventional TACE, respectively, resulting in a HR of 0.59 (95\% CI: 0.4-0.87, $p=0.006$ ).

Consensus-based statement 12: For patients with unresectable early and mid-stage HCC, TACE combined with sorafenib is a treatment option since it can improve PFS (grade 3 recommendation, evidence level 2).

For those patients with good liver functions, TACE is appropriate therapy. Several retrospective studies and meta-analyses have shown that TACE combined with sorafenib improved clinical outcomes compared to TACE treatment alone [61-63]. The systematic review and meta-analysis reported by Zhang et al. [63] showed that TACE combined with sorafenib increased ORR (OR 3.59, 95\% CI: $\left.1.74-7.39 ; I^{2}=21 \%, p=0.001\right)$ and the disease control rate (OR 4.72, 95\% CI: $1.75-12.72 ; I^{2}=56 \%, p=$ 0.002) compared to TACE treatment alone in patients who had portal vein tumor thrombosis and in addition improved the OS at 6 months and 1 year (HR 0.62; 95\% CI: $0.51-0.75 ; I^{2}=30 \%, p<0.000$ ).

Qiu and colleagues [64] retrospectively analyzed 251 cases of advanced HCC with extrahepatic metastases treated with various TACE-based combinations including TACE/sorafenib and TACE/apatinib. It was demonstrated that adding molecular targeted therapies to TACE provided better outcomes compared to TACE treatment alone. Furthermore, it was found that TACE combined with sorafenib prolonged TTP $(2.7$ months vs. 2.1 months, $p=0.011)$ [65] but that OS (9.1 months vs. 6.7 months, $p$ $=0.210$ ) was not significantly different to TACE therapy alone in patients with $\mathrm{HCC}$ with portal vein tumor thrombosis and/or extrahepatic metastases. Nonetheless, to clarify these conflicting results and to determine the value of TACE combinations, prospective phase 3 studies should be performed [66].

Consensus-based statement 13: For some patients with advanced HCC with good liver function but exhibiting extrahepatic metastasis, TACE as an adjunct to molecular targeted drug therapy is an option (grade 3 recommendation, evidence level 4).

Hepatic artery infusion chemotherapy (HAIC), as another interventional therapy, has also been investigated in patients with advanced stage HCC, complicated by tumor thrombosis of the portal vein [67]. The multicenter Japanese SILIUS randomized phase 3 study enrolled 206 patients [68]. SILIUS compared HAIC (low-dose cisplatin and 5-FU chemotherapy regimen) combined with sorafenib versus sorafenib therapy alone. The median OS time was 11.8 months (9.1-14.5 months) for HAIC/ sorafenib therapy and 11.5 months (95\% CI: 18.2-14.8) for sorafenib alone, respectively, resulting in a $\mathrm{HR}$ of 
1.009 (95\% CI: $10.743-1.371, p=0.955)$ but did not reach a statistically significant difference compared to the control study groups. However, analyzing the data separately with regard to the extent of portal vein tumor thrombosis, the median OS time was 11.4 months (95\% CI: 7.0-15.9 months) for the combination and 6.5 months (95\% CI: 4.5-8.4 months) for sorafenib alone in cases when tumor thrombosis involved the main portal vein were statistically significantly different (HR 0.493, 95\% CI: 0.240 $1.014 ; p=0.050)$. SILIUS also showed an improved TTP, favoring significantly the combination (5.3 months, $95 \%$ CI: 3.9-6.7 months) compared to sorafenib alone (3.5 months, 95\% CI: $2.5-4.6$ months), resulting in a HR of 0.645 (95\% CI: $0.477-0.872, p=0.004$ ).

Another multicenter phase 3 study which enrolled Chinese patients confirmed the superiority of the HAIC combination [69]. OS and PFS were improved by FOLFOXHAIC plus sorafenib versus sorafenib alone in 247 patients with advanced HCC and portal vein tumor thrombosis, with Vp3-Vp4 predominance (80.6\%). Sixteen patients treated with HAIC/sorafenib and 1 patient with sorafenib alone became resectable. Median OS was 13.37 months for the HAIC-based combination and 7.13 months for sorafenib alone, respectively, resulting in a HR of 0.35 (95\% CI: $0.26-0.48, p<0.010$ ). PFS was 7.03 months (95\% CI: 6.05-8.02 months) and 2.6 months (95\% CI: 2.15-3.05 months) with a HR of 0.33 (95\% CI: $0.25-0.43$, $p<0.001)$. In the subgroup analysis, the outcome by extend portal vein tumor thrombosis also favored FOLFOXHAIC/sorafenib with a median OS for Vp1-2: 18.17 months (95\% CI: 11.19-25.15) versus 10.87 months (95\% CI: 6.79-14.95, $p=0.002)$; Vp3: 13.47 months (95\% CI: 9.83-17.1) versus 6.27 months (95\% CI: 4.81-7.73, $p<$ 0.001 ); and Vp4: 9.47 months (95\% CI: 5.97-12.96) versus 5.5 months (95\% CI: 3.62-7.38, $p<0.001$ ).

Consensus-based statement 14: For advanced HCC with good liver functions and portal vein tumor thrombosis, especially for patients with major involvement of the portal vein, FOLFOX HAIC combined with sorafenib may be an option (grade 1 recommendation, evidence level 1). TACE combined with molecular targeted therapy is another option (grade 2 recommendation, evidence level 4).

\section{Molecular Targeted Drug Dose Selection and AE \\ Management \\ Dose Selection}

A number of phase 3 and post-marketing real-world studies have shown that standard doses of sorafenib were well tolerated $[7,8,67,70]$, and it was recommended to start with a sorafenib standard dose. However, for lenva- tinib, the incidence of treatment-related treatment-emergent AEs of grade $\geq 3$ seemed to have been higher than that of sorafenib (57\% vs. $49 \%$ ) in the REFLECT study [9]. High rates of lenvatinib dose reductions and discontinuations have been observed in several other studies mainly due to thyroid dysfunction, fatigue, and appetite loss as well as albumin-bilirubin grade 2 [71, 72], while relative dose intensities (RDIs) of $\geq 75 \%$ for 8 weeks [73] and $\geq 70 \%$ for 4 weeks $[74,75]$ were noted to be factors for favorable responses of lenvatinib therapies for HCC.

The toxicity profile of regorafenib in HCC is similar to that seen for advanced colorectal cancer [13]. Hand-footskin reactions and fatigue that occur during the early phase of therapy may lead to the early termination of regorafenib. Results of a number of phase 2 studies have shown that an appropriate reduction of the initial dose in the first cycle and adjustment to the standard dose in the second and subsequent cycles did not reduce efficacy of regorafenib but were less toxic in treating advanced colorectal cancer $[69,76,77]$. Nonetheless, reliable real-world data are yet not available for advanced HCC in China. In Japan and South Korea, however, clinical practice seems to generally adjust the second-line setting of regorafenib initially to $120 \mathrm{mg}$ or $80 \mathrm{mg}$ for patients with Child-Pugh grade $\mathrm{B}$ or $\mathrm{C}$ liver functions, renal insufficiency, body weight $<50 \mathrm{~kg}$, or with grade 2-3 TRAEs during first-line sorafenib therapy $[78,79]$. OS and PFS times however were significantly longer in patients who received a $\geq 50 \%$ RDI of regorafenib in the first month compared to patients who received a $<50 \% \mathrm{RDI}[80]$. Clinically relevant toxicity data obtained from the prospective large scale real-world REFINE study were reported at ASCO 2020. Patients were enrolled (500), mainly having been pretreated with sorafenib, with the majority having a PS score of $>1$ and a liver function of Child-Pugh stage B. Patients were given a standard dose of $160 \mathrm{mg}$ regorafenib in $57 \%, 120 \mathrm{mg}$ in $13 \%, 80 \mathrm{mg}$ in $28 \%$, and $40 \mathrm{mg}$ in $2 \%$ of them as starting medication, respectively. The incidence of TRAEs was $66 \%$, and of grades $\geq 3$ was $21 \%$, which was lower than in phase 3 studies ( $93 \%$ and 50\%, respectively). Seventy five percent of patients developed TRAEs during the first 8 weeks of treatment, and $83 \%$ underwent dose reduction. The median PFS was similar to that of the RESORCE study (3.7 months vs. 3.1 months), and the median OS was longer than in the RESORCE study (13.2 months vs. 10.6 months) [81].

Consensus-based statement 15: For patients candidates for first-line molecular targeted therapy the standard recommended dose should be used as a starting dose (grade 1 recommendation, evidence level 1). Patients with Child- 
Pugh grade B or $C$ liver functions, renal insufficiency, a body weight $<50 \mathrm{~kg}$, or with grade 2-3 TRAEs during sorafenib, the initial dose of regorafenib in the second-line setting should be adjusted to $120 \mathrm{mg}$ or $80 \mathrm{mg}$ (grade 3 recommendation, evidence level 3).

TRAEs of Molecular Targeted Therapies

For molecular targeted drugs used to treat HCC, common AEs include fatigue, diarrhea, hand-foot-skin reactions, hypertension, anorexia, weight loss, proteinuria, nausea, vomiting, hoarseness, hypoproteinemia, anemia, a decrease in platelet counts and white blood cell numbers, and abnormal liver functions. Life-threatening TRAEs included congestive heart failure, myocardial ischemia or myocardial infarction, a hypertensive crisis, gastrointestinal perforation/bleeding, and airway and intracranial hemorrhage. The incidence of TRAEs under the targeted drugs has been $61.0 \%$ in a recent retrospective Chinese study [82]. Treatment modifications, such as drug suspension, dose reduction, or permanent withdrawal should be undertaken according to the severity of TRAEs. Before initiating therapies, patients should be informed regarding the potential occurrence of TRAEs and appropriate preventative measures taken including exercise, skin ointment, diet, and blood pressure monitoring, as well as doctor consultations.

Consensus-based statement 16: For TRAEs, general principles of pre-medication should be obeyed during and between therapies with respect to potential hand-foot-skin reactions, hypertension, diarrhea, and to other group toxicities of molecular targeted therapies. Adequate treatment modifications should be initiated such as treatment delay, dose reduction or withdrawal of medication. For serious TRAES, interdisciplinary advice is critical (grade 1 recommendation, evidence level 1).

Use of Traditional Chinese Medicine to Manage

Drug Toxicities

Traditional Chinese medicine (TCM) can be beneficial in treating skin and mucosal AEs. External washing and treating hand-foot-skin toxicities with TCM compounds may reverse local heat, resolve dampness, improve the blood microcirculation, and remove micro-thrombosis, resulting in a response rate of $80-95 \%$. It also helps in the treatment of rash and mucositis, maintaining drug dosage and avoiding treatment withdrawal [83]. TCM may be appropriate in alleviating treatment-related diarrhea. Since TCM compounds sooth the liver, tonify the spleen, clear dampness, and promote diuresis, they can be effective in relieving diarrhea. Studies also suggest that TCM

Chinese Expert Statements on Molecular

Targeted Therapy for HCC may synergistically increase the antitumor effect of molecular targeted drugs [84], thus providing another rationale for combining oral TCM with molecular targeted therapies. In addition, TCM and acupuncture are commonly used to relieve cancer-related fatigue $[85,86]$.

Consensus-based statement 17: TCM can be used to treat molecular targeted drug-related skin and mucosal toxicity and molecular targeted drug-related diarrhea (grade 1 recommendation, evidence level 2).

Consensus-based statement 18: TCM has definite efficacy in treating cancer-related fatigue, but for fatigue caused by molecular targeted therapy patients should be first introduced to clinical trial programs (grade 2 recommendation, evidence level 1).

\section{Efficacy Evaluation of Molecular Targeted Therapies}

Evidence

RECIST 1.1 guidelines are used internationally to measure the treatment effect produced by conventional chemotherapy of solid malignant tumors. But, today, commonly applied molecular targeted drugs such as sorafenib and lenvatinib display their antitumor effects mainly by inhibiting tumor angiogenesis. CT or MRI, apart from pure volume reduction, usually reveals an impact on arterial blood flow, tumor necrosis, and liquefaction in tumors. Additionally, due to tumor necrosis after local treatments such as ablation, embolization, and radiotherapy, CT/MRI images may look unaltered or even show an increased tumor volume. Bearing this in mind, RECIST, which is based essentially on the maximum tumor diameter, may have shortcomings in measuring the actual efficacy of molecular targeted therapies and may not reflect specific response patterns.

Therefore, in 2009, the American Association for the Study of Liver Diseases proposed mRECIST for a comprehensive evaluation of selective HCC treatments using $\mathrm{CT} / \mathrm{MRI}$ measurements in the arterial phase of the maximum diameter of the enhanced region within a tumor. Studies have shown that ORR assessed by mRECIST is an independent prognostic factor for molecular targeted therapies of HCC. Comparing RECIST with mRECIST in the efficacy evaluation of sorafenib, ORR increased from $6.5 \%$ to $9.2 \%$ and up to $17.5 \%[68,87]$. The OS of patients responding to lenvatinib or sorafenib was prolonged by up to 22.4 months, 11 months more than for nonresponders $(p<0.001)$ [88]. In the case of local treatment using mRECIST, responders compared to nonresponders had a $61 \%$ decreased risk of death $(p<0.000)$ [89], and such defined responders had a better prognosis than those assessed by RECIST [90].

Liver Cancer 2022;11:192-208 
Table 4. Comparison of RECIST 1.1 and mRECIST

\begin{tabular}{lll}
\hline $\begin{array}{l}\text { Response in target } \\
\text { lesions }\end{array}$ & RECIST 1.1 & mRECIST \\
\hline Complete response & Disappearance of all target lesions & $\begin{array}{l}\text { Disappearance of any intratumoral arterial enhancement in all } \\
\text { target lesions }\end{array}$ \\
\hline Partial response & $\begin{array}{l}\text { At least a 30\% decrease in the sum of diameters of target } \\
\text { lesions, taking as a reference the baseline sum of the } \\
\text { diameters of the target lesions }\end{array}$ & $\begin{array}{l}\text { At least a 30\% decrease in the sum of diameters of viable } \\
\text { (enhancement in the arterial phase) target lesions, taking as the } \\
\text { reference the baseline sum of the diameters of the target lesions }\end{array}$ \\
\hline Stable disease & $\begin{array}{l}\text { Any case that did not qualify for either partial response or } \\
\text { progressive disease }\end{array}$ & $\begin{array}{l}\text { Any case that do not qualify for either partial response or } \\
\text { progressive disease }\end{array}$ \\
\hline Progressive disease & $\begin{array}{l}\text { An increase } \geq 20 \% \text { in the sum of the diameters of target } \\
\text { lesions, taking as the reference the smallest sum of the } \\
\text { diameters of the target lesions recorded since treatment } \\
\text { started }\end{array}$ & $\begin{array}{l}\text { An increase of } \geq 20 \% \text { in the sum of the diameters of viable } \\
\text { (enhancing) target lesions, taking as the reference the smallest } \\
\text { sum of the diameters of viable (enhancing) target lesions } \\
\text { recorded since treatment started }\end{array}$ \\
\hline
\end{tabular}

Lymph nodes identified as target lesions should always have the actual short-axis measurement recorded.

In summary, mRECIST compared to RECIST was better able to reflect short-term efficacy and may also have an advantage in predicting long-term survival and thus is a promising guiding factor for clinical practice in evaluating the efficacy of molecular targeted therapy for HCC. It is recommended for the selection of target lesions to avoid necrotic areas and to choose instead enhanced areas in the arterial phase which can be repeatedly measured. In general, portal vein tumor thrombosis and ascites should not be regarded as target lesions but can be followed up as nontarget lesions.

Consensus-based statement 19: RECIST 1.1 are often used for efficacy evaluation in HCC patients under molecular targeted therapies. One option comprises the $m R E$ CIST, reflecting short-term efficacy better and should be followed in combination with RECIST 1.1 (grade 1 recommendation, evidence level 1) (Table 4).

\section{Predictors in Using Molecular Targeted Therapies}

HCC markers are mainly used for diagnoses. The clarification of their predictive values for molecular targeted treatment, in particular with sorafenib, is under investigation. TRAES to sorafenib correlate with the prognosis of patients with HCC, most prominently with hand-foot-skin toxicities. A recent meta-analysis involving 1,017 patients treated with sorafenib found that hand-foot-skin reactions correlated significantly with efficacy and TTP [91]. In addition, a retrospective analysis based on SHARP and ORIENTAL phase 3 studies showed that sorafenib was more effective in treating HCV-related to HCC compared to patients infected with hepatitis B virus, probably because of inflammation caused by persistent replication of the virus [92]. Patients suffering from a hepatitis $B$ infection in general received antiviral treatment, leading to low virus replication.

In terms of serological markers, patients receiving sorafenib whose AFP level dropped by more than $20 \%$ within 6-8 weeks of treatment had better outcomes and prognosis. An early decline of the AFP level resulted in a higher ORR (33\% vs. $8 \%$ ) and longer OS (15.3 months vs. 4.1 months) [93]. The SHARP and ORIENTAL studies found that patients with a lower peripheral blood neutrophil to lymphocyte ratio had a longer OS and generally were more likely to benefit from sorafenib [92], probably reflecting the inflammation state and correlation between pro-tumor inflammation and the antitumor immune response. Nevertheless, it can be argued that no definition standard yet exists for the NLR-threshold and that prediction value need further clarification.

Another study conducted in Japan found that increased circulating fibroblast growth factor 19 and decreased angiopoietin-2 serum concentrations exhibited in combination an independent association with PFS under lenvatinib therapy of HCC patients [94]. Another Chinese study suggested that fibroblast growth factor receptor 4 expression levels and Treg infiltration in tumors might serve as biomarkers for screening HCC patients, who would be suitable for lenvatinib plus anti-PD-1 combination therapies [95]. For anlotinib, the $\mathrm{C}-\mathrm{X}-\mathrm{C}$ motif chemokine ligand 1 (CXCL1) plasma concentration has been proposed to be an efficacy predictor for HCC treatments [12]. 
Consensus-based statement 20: For patients with HCC likely to be treated with sorafenib, hepatitis virus status and peripheral blood neutrophil/lymphocyte ratio are optional factors to be included in the treatment prediction (grade 3 recommendation, evidence level 4).

Consensus-based statement 21: The grade of TRAES and the change of AFP serum level are also optional treatment predictors (grade 3 recommendation, evidence level 4).

Consensus-based statement 22: Serum markers might serve as prognostic indicators for TKI treatments or as selection tools for TKI combination treatments (grade 3 recommendation, evidence level 3).

\section{Role of Multidisciplinary Teams}

A comprehensive diagnosis and treatment concept for malignant tumors has become a critical issue. The existence of a multidisciplinary team (MDT) helps to overcome the limitations of single disciplinary decisions to guarantee broader knowledge and experience based case discussions and finally evidence-based individualized diagnosis, treatment, and follow-up concepts. HCC is a heterogeneous neoplasm, differing in its origins and biological nature. These factors underline the importance of MDT, which only can provide the required evidencebased comprehensive recommendations on tumor characteristics and the clinical stage, molecular pathological diagnosis, and treatment options. Patients suffering from mid-stage and advanced HCC often require multidisciplinary therapies, which combine local and systemic treatment options [96]. More than 90\% of HCC patients have underlying chronic liver disease or cirrhosis and because of the toxicity risk of tumor therapies, a liver specialist or gastroenterologist should definitely be a part of HCC-MDT [97]. Needless to say, drug-related toxicity may require expert assistance from various clinical disciplines such as hepatology, dermatology, and TCM.

Consensus-based statement 23: For the determination of evidence based treatment strategies in HCC, MDT should definitely be established in any centers dealing with liver cancer patients (grade 1 recommendation, evidence level 1).

\section{Conclusions}

In recent years, the treatment of liver cancer has undergone rapid changes, and many therapeutic drugs and new therapeutic technologies have emerged. However, targeted antiangiogenic drugs remain the backbone of systemic treatment for advanced HCC, although atezolizumab combined with bevacizumab has become a new option for the first-line treatment of advanced HCC.

While looking forward to the development of more antiangiogenic drugs with low toxicity and high efficacy, it is an important direction for liver cancer therapy to explore continuously other therapeutic targets in basic research and to find more specific targeted drugs. At the same time, on the basis of targeted drugs, how to effectively integrate the existing and emerging new therapeutic technologies is a major challenge. In the above chapter, we discussed recent research evidence and clinical diagnosis and treatment experience to provide consensus statements. We also look forward to carrying out more researcher-initiated multidisciplinary studies to provide more evidence-based proposals for the optimization of targeted therapy-based advanced liver cancer treatments.

\section{Conflict of Interest Statement}

Jia Fan is an editorial board member of Liver Cancer. The remaining authors have no conflicts of interest to declare.

\section{Funding Sources}

This study was supported by the State Key Project on Infection Diseases of China (grant no. 2017ZX10201021-007-003); the CAMS Innovation Fund for Medical Sciences (CIFMS) (grant no. 2017-12M-4-002); the Non-profit Central Research Institution Fund of Chinese Academy of Medical Sciences (grant no. 2019PT310026); the Sanming Project of Medicine in Shenzhen (grant no. SZSM202011010); and the Capital Health Research and Development of Special (grant no. 2018-1-4021). The funders had no role in the design of the study and collection, analysis, and interpretation of data or in writing the manuscript.

\section{Author Contributions}

Jianqiang Cai served as the principal guidance for this consensus. All the authors contributed to the preparation of the paper and have read and approved the final manuscript for publication.

\section{Data Availability Statement}

Data sharing is not applicable to this article as no datasets were generated or analyzed during the current study. 


\section{References}

1 Zheng R, Sun K, Zhang S, Zeng H, Zou X, Chen R, et al. Analysis of the prevalence of malignant tumors in China in 2015. Zhong Hua Zhong Liu Za Zhi. 2019;41(1):19-28.

2 Huang A, Yang XR, Chung WY, Dennison AR, Zhou J. Targeted therapy for hepatocellular carcinoma. Signal Transduct Target Ther. 2020;5(1):146.

3 Yang C, Qin S. Apatinib targets both tumor and endothelial cells in hepatocellular carcinoma. Cancer Med. 2018;7(9):4570-83.

4 Yau T, ParkJW, Finn RS, Cheng AL, Mathurin P, Edeline J, et al. LBA38_PR - checkmate 459: a randomized, multi-center phase III study of nivolumab (NIVO) vs sorafenib (SOR) as first-line (1L) treatment in patients (pts) with advanced hepatocellular carcinoma (aHCC). Ann Oncol. 2019;30:v874-75.

5 Finn RS, Ryoo BY, Merle P, Kudo M, Bouattour M, Lim HY, et al. Pembrolizumab as second-line therapy in patients with advanced hepatocellular carcinoma in KEYNOTE-240: a randomized, double-blind, phase III trial. J Clin Oncol. 2020;38(3):193-202.

6 Vogel A, Cervantes A, Chau I, Daniele B, Llovet JM, Meyer T, et al. Hepatocellular carcinoma: ESMO clinical practice guidelines for diagnosis, treatment and follow-up. Ann Oncol. 2018;29(Suppl 4):iv238-55.

7 Llovet JM, Ricci S, Mazzaferro V, Hilgard P, Gane E, Blanc JF, et al. Sorafenib in advanced hepatocellular carcinoma. $\mathrm{N}$ Engl J Med. 2008;359(4):378-90.

8 Cheng AL, Kang YK, Chen Z, Tsao CJ, Qin S, Kim JS, et al. Efficacy and safety of sorafenib in patients in the Asia-Pacific region with advanced hepatocellular carcinoma: a phase III randomised, double-blind, placebo-controlled trial. Lancet Oncol. 2009;10(1):25-34.

9 Kudo M, Finn RS, Qin S, Han KH, Ikeda K, Piscaglia F, et al. Lenvatinib versus sorafenib in first-line treatment of patients with unresectable hepatocellular carcinoma: a randomised phase 3 non-inferiority trial. Lancet. 2018;391(10126):1163-73.

10 Bi F, Qin S, Gu S, Bai Y, Chen Z, Wang Z, et al. Donafenib versus sorafenib as first-line therapy in advanced hepatocellular carcinoma: an open-label, randomized, multicenter phase II/III trial. J Clin Oncol. 2020;38(15_ Suppl):4506.

11 Qin S, Bai Y, Ouyang X, Cheng Y, Li J, Xu J, et al. Apatinib for patients with advanced hepatocellular carcinoma: a randomised, openlabel, multicentre,phase II clinical trial. Lin Chuang Zhong Liu Xue Za Zhi. 2017;22(12): 1057-65.

12 Sun Y, Zhou A, Zhang W, Jiang Z, Chen B, Zhao J, et al. Anlotinib in the treatment of advanced hepatocellular carcinoma: an openlabel phase II study (ALTER-0802 study). Hepatol Int. 2021;15(3):621-9.
13 Bruix J, Qin S, Merle P, Granito A, Huang YH, Bodoky G, et al. Regorafenib for patients with hepatocellular carcinoma who progressed on sorafenib treatment (RESORCE): a randomised, double-blind, placebo-controlled, phase 3 trial. Lancet. 2017;389(10064):56-66.

14 Li Q, Qin S, Gu S, Chen X, Lin L, Wang Z, et al. Apatinib as second-line therapy in Chinese patients with advanced hepatocellular carcinoma: a randomized, placebo-controlled, double-blind, phase III study. J Clin Oncol. 2020;38(15_Suppl):4507.

15 Abou-Alfa GK, Meyer T, Cheng AL, ElKhoueiry AB, Rimassa L, Ryoo BY, et al. Cabozantinib in patients with advanced and progressing hepatocellular carcinoma. N Engl J Med. 2018;379(1):54-63.

16 Zhu AX, Kang YK, Yen CJ, Finn RS, Galle PR, Llovet JM, et al. Ramucirumab after sorafenib in patients with advanced hepatocellular carcinoma and increased $\alpha$-fetoprotein concentrations (REACH-2): a randomised, doubleblind, placebo-controlled, phase 3 trial. Lancet Oncol. 2019;20(2):282-96.

17 Finn RS, Qin S, Ikeda M, Galle PR, Ducreux M, Kim T-Y, et al. IMbrave150: updated overall survival (OS) data from a global, randomized, open-label phase III study of atezolizumab (atezo) + bevacizumab (bev) versus sorafenib (sor) in patients (pts) with unresectable hepatocellular carcinoma (HCC). J Clin Oncol. 2021;39(3_Suppl):267.

18 Ren Z, Xu J, Bai Y, Xu A, Cang S, Du C, et al. Sintilimab plus a bevacizumab biosimilar (IBI305) versus sorafenib in unresectable hepatocellular carcinoma (ORIENT-32): a randomised, open-label, phase 2-3 study. Lancet Oncol. 2021;22(7):977-90.

19 Finn RS, Ikeda M, Zhu AX, Sung MW, Baron $\mathrm{AD}$, Kudo M, et al. Phase Ib study of lenvatinib plus pembrolizumab in patients with unresectable hepatocellular carcinoma. J Clin Oncol. 2020;38(26):2960-70.

$20 \mathrm{Xu} \mathrm{J}$, Shen J, Gu S, Zhang Y, Wu L, Wu J, et al. Camrelizumab in combination with apatinib in patients with advanced hepatocellular carcinoma (RESCUE): a nonrandomized, openlabel, phase II trial. Clin Cancer Res. 2021; 27(4):1003-11.

21 Xu J, Zhang Y, Jia R, Yue C, Chang L, Liu R, et al. Anti-PD-1 antibody SHR-1210 combined with apatinib for advanced hepatocellular carcinoma, gastric, or esophagogastric junction cancer: an open-label, dose escalation and expansion study. Clin Cancer Res. 2019;25(2):515-23.

22 El-Khoueiry AB, Kim RD, Harris WP, Sung MW, Waldschmidt D, Iqbal S, et al. Phase Ib study of regorafenib (REG) plus pembrolizumab (PEMBRO) for first-line treatment of advanced hepatocellular carcinoma (HCC). J Clin Oncol. 2020;38(4_Suppl):564.
23 Kudo M, Ikeda M, Motomura K, Okusaka T, Kato N, Dutcus CE, et al. A phase Ib study of lenvatinib (LEN) plus nivolumab (NIV) in patients (pts) with unresectable hepatocellular carcinoma (uHCC): study 117. J Clin Oncol. 2020;38(4_Suppl):513.

24 El-Khoueiry AB, Kim RD, Harris WP, Sung MW, Waldschmidt D, Cabrera R, et al. Updated results of a phase $1 \mathrm{~b}$ study of regorafenib (REG) $80 \mathrm{mg} /$ day or $120 \mathrm{mg} /$ day plus pembrolizumab (PEMBRO) for first-line treatment of advanced hepatocellular carcinoma (HCC). J Clin Oncol. 2021;39(15_Suppl):4078.

25 Zhu AX, Rosmorduc O, Evans TR, Ross PJ, Santoro A, Carrilho FJ, et al. SEARCH: a phase III, randomized, double-blind, placebo-controlled trial of sorafenib plus erlotinib in patients with advanced hepatocellular carcinoma. J Clin Oncol. 2015;33(6):559-66.

26 Abou-Alfa GK, Johnson P, Knox JJ, Capanu M, Davidenko I, Lacava J, et al. Doxorubicin plus sorafenib vs doxorubicin alone in patients with advanced hepatocellular carcinoma: a randomized trial. JAMA. 2010;304(19): 2154-60.

27 Srimuninnimit V, Sriuranpong V, Suwanvecho S. Efficacy and safety of sorafenib in combination with gemcitabine in patients with advanced hepatocellular carcinoma: a multicenter, open-label, single-arm phase II study. Asia Pac J Clin Oncol. 2014;10(3):25560.

28 Petrini I, Lencioni M, Ricasoli M, Iannopollo M, Orlandini C, Oliveri F, et al. Phase II trial of sorafenib in combination with 5-fluorouracil infusion in advanced hepatocellular carcinoma. Cancer Chemother Pharmacol. 2012; 69(3):773-80.

29 Liu Y, Yue H, Xu S, Wang F, Ma N, Li K, et al. First-line gemcitabine and oxaliplatin (GEMOX) plus sorafenib, followed by sorafenib as maintenance therapy, for patients with advanced hepatocellular carcinoma: a preliminary study. Int J Clin Oncol. 2015;20(5):9529.

30 Bruix J, Takayama T, Mazzaferro V, Chau GY, Yang J, Kudo M, et al. Adjuvant sorafenib for hepatocellular carcinoma after resection or ablation (STORM): a phase 3, randomised, double-blind, placebo-controlled trial. Lancet Oncol. 2015;16(13):1344-54.

31 Wang SN, Chuang SC, Lee KT. Efficacy of sorafenib as adjuvant therapy to prevent early recurrence of hepatocellular carcinoma after curative surgery: a pilot study. Hepatol Res. 2014;44(5):523-31

32 Zhang XP, Chai ZT, Gao YZ, Chen ZH, Wang $\mathrm{K}$, Shi J, et al. Postoperative adjuvant sorafenib improves survival outcomes in hepatocellular carcinoma patients with microvascular invasion after R0 liver resection: a propensity score matching analysis. HPB. 2019;21(12): 1687-96. 
33 Liao Y, Zheng Y, He W, Li Q, Shen J, Hong J, et al. Sorafenib therapy following resection prolongs disease-free survival in patients with advanced hepatocellular carcinoma at a high risk of recurrence. Oncol Lett. 2017;13(2): 984-92.

34 Zhuang L, Wen T, Xu M, Yang J, Wang W, $\mathrm{Wu} \mathrm{H}$, et al. Sorafenib combined with hepatectomy in patients with intermediate-stage and advanced hepatocellular carcinoma. Arch Med Sci. 2017;13(6):1383-93.

35 Huang Y, Cheng X, Sun P, Li T, Song Z, Zheng Q. Supplementary sorafenib therapies for hepatocellular carcinoma-A systematic review and meta-analysis: supplementary sorafenib for liver cancer. J Clin Gastroenterol. 2019; 53(7):486-94.

36 Chen J-H, Lu L, Wen T-F, Huang Z-Y, Zhang $\mathrm{T}$, Zeng $\mathrm{Y}-\mathrm{Y}$, et al. Adjuvant lenvatinib in combination with TACE for hepatocellular carcinoma patients with high risk of postoperative relapse (LANCE): interim results from a muticenter prospective cohort study. J Clin Oncol. 2020;38(15_Suppl):4580.

37 Waghray A, Balci B, El-Gazzaz G, Kim R, Pelley R, Narayanan Menon KV, et al. Safety and efficacy of sorafenib for the treatment of recurrent hepatocellular carcinoma after liver transplantation. Clin Transplant. 2013;27(4): $555-61$.

38 de'Angelis N, Landi F, Carra MC, Azoulay D. Managements of recurrent hepatocellular carcinoma after liver transplantation: a systematic review. World J Gastroenterol. 2015; 21(39):11185-98

39 de'Angelis N, Landi F, Nencioni M, Palen A, Lahat E, Salloum C, et al. Role of sorafenib in patients with recurrent hepatocellular carcinoma after liver transplantation. Prog Transplant. 2016;26(4):348-55.

40 Kang SH, Cho H, Cho EJ, Lee JH, Yu SJ, Kim YJ, et al. Efficacy of sorafenib for the treatment of post-transplant hepatocellular carcinoma recurrence. J Korean Med Sci. 2018; 33(45):e283.

41 Chagas AL, Felga GEG, Diniz MA, Silva RF, Mattos AA, Silva R, et al. Hepatocellular carcinoma recurrence after liver transplantation in a Brazilian multicenter study: clinical profile and prognostic factors of survival. Eur J Gastroenterol Hepatol. 2019;31(9):1148-56.

42 Iavarone $M$, Invernizzi $F$, Czauderna $C$, Sanduzzi-Zamparelli M, Bhoori S, Amaddeo G, et al. Preliminary experience on safety of regorafenib after sorafenib failure in recurrent hepatocellular carcinoma after liver transplantation. Am J Transplant. 2019;19(11):317684.

43 Vivarelli M, Cucchetti A, La Barba G, Ravaioli M, Del Gaudio M, Lauro A, et al. Liver transplantation for hepatocellular carcinoma under calcineurin inhibitors: reassessment of risk factors for tumor recurrence. Ann Surg. 2008;248(5):857-62.

44 Organ Transplantation Physician Branch of Chinese Medical Doctor Association (OTBoCMA). The Chinese clinical practice guideline on liver transplantation for hepatocellular carcinoma, 2018. Lin Chuang Gan Dan Bing Za Zhi. 2019;35(2):275-80.

45 Jung DH, Tak E, Hwang S, Song GW, Ahn CS, $\mathrm{Kim} \mathrm{KH}$, et al. Antitumor effect of sorafenib and mammalian target of rapamycin inhibitor in liver transplantation recipients with hepatocellular carcinoma recurrence. Liver Transpl. 2018;24(7):932-45.

46 Invernizzi F, Iavarone M, Zavaglia C, Mazza S, Maggi U, Cesarini L, et al. Experience with early sorafenib treatment with mTOR inhibitors in hepatocellular carcinoma recurring after liver transplantation. Transplantation. 2020;104(3):568-74.

47 Huang L, Li GM, Zhu JY, Li Z, Li T, Leng XS. Efficacy of sorafenib after liver transplantation in patients with primary hepatic carcinoma exceeding the Milan criteria: a preliminary study. Onco Targets Ther. 2012;5:457-62.

48 Shetty K, Dash C, Laurin J. Use of adjuvant sorafenib in liver transplant recipients with high-risk hepatocellular carcinoma. J Transplant. 2014;2014:913634.

49 Siegel AB, El-Khoueiry AB, Finn RS, Guthrie KA, Goyal A, Venook AP, et al. Phase I trial of sorafenib following liver transplantation in patients with high-risk hepatocellular carcinoma. Liver Cancer. 2015;4(2):115-25.

50 Bureau of Medical Administration, National Health Commission of the People's Republic of China. Guidelines for diagnosis and treatment of primary liver cancer in China (2019 edition). Lin Chuang Gan Dan Bing Za Zhi. 2020;7(1):5-23

51 Ni JY, Sun HL, Luo JH, Jiang XY, Chen D, Wang WD, et al. Transarterial chemoembolization and sorafenib combined with microwave ablation for advanced primary hepatocellular carcinoma: a preliminary investigation of safety and efficacy. Cancer Manag Res. 2019;11:9939-50

52 Chen SW, Lin LC, Kuo YC, Liang JA, Kuo CC, Chiou JF. Phase 2 study of combined sorafenib and radiation therapy in patients with advanced hepatocellular carcinoma. Int $J$ Radiat Oncol Biol Phys. 2014;88(5):1041-7.

53 Zhao Y, Zhu X, Wang H, Dong D, Gao S, Zhu $\mathrm{X}$, et al. Safety and efficacy of transcatheter arterial chemoembolization plus radiotherapy combined with sorafenib in hepatocellular carcinoma showing macrovascular invasion. Front Oncol. 2019;9:1065.

54 Kim BK, Kim DY, Byun HK, Choi HJ, Beom $\mathrm{SH}$, Lee HW, et al. Efficacy and safety of liverdirected concurrent chemoradiotherapy and sequential sorafenib for advanced hepatocellular carcinoma: a prospective phase 2 trial. Int $J$ Radiat Oncol Biol Phys. 2020;107(1):106-15.

55 Nomura T, Tani J, Deguchi A, Nakahara M, Oura K, Tadokoro T, et al. Efficacy of combined modality therapy with sorafenib following hepatic arterial injection chemotherapy and three-dimensional conformal radiotherapy for advanced hepatocellular carcinoma with major vascular invasion. Mol Clin Oncol. 2019;11(5):447-54.
56 Yin X, Xu Q. Observation of the curative effect of Sorafenib combined stereotactic body radiation therapy in the treatment of primary hepatocellular carcinoma with pulmonary metastasis. Zhong Guo Lin Chuang Yi Xue. 2016;23(5):618-24.

57 Sun T, He J, Zhang S, Sun J, Zeng M, Zeng Z. Simultaneous multitarget radiotherapy using helical tomotherapy and its combination with sorafenib for pulmonary metastases from hepatocellular carcinoma. Oncotarget. 2016; 7(30):48586-99.

58 Lencioni R, Llovet JM, Han G, Tak WY, Yang J, Guglielmi A, et al. Sorafenib or placebo plus TACE with doxorubicin-eluting beads for intermediate stage HCC: the SPACE trial. J Hepatol. 2016;64(5):1090-8.

59 Meyer T, Fox R, Ma YT, Ross PJ, James MW, Sturgess R, et al. Sorafenib in combination with transarterial chemoembolisation in patients with unresectable hepatocellular carcinoma (TACE 2): a randomised placebo-controlled, double-blind, phase 3 trial. Lancet Gastroenterol Hepatol. 2017;2(8):565-75.

60 Kudo M, Ueshima K, Ikeda M, Torimura T, Tanabe N, Aikata H, et al. Randomised, multicentre prospective trial of transarterial chemoembolisation (TACE) plus sorafenib as compared with TACE alone in patients with hepatocellular carcinoma: TACTICS trial. Gut. 2020;69(8):1492-501.

$61 \mathrm{Qu}$ XD, Chen CS, Wang JH, Yan ZP, Chen JM, Gong GQ, et al. The efficacy of TACE combined sorafenib in advanced stages hepatocellullar carcinoma. BMC Cancer. 2012;12: 263.

62 Zhu K, Chen J, Lai L, Meng X, Zhou B, Huang $\mathrm{W}$, et al. Hepatocellular carcinoma with portal vein tumor thrombus: treatment with transarterial chemoembolization combined with sorafenib: a retrospective controlled study. Radiology. 2014;272(1):284-93.

63 Zhang X, Wang K, Wang M, Yang G, Ye X, $\mathrm{Wu} \mathrm{M}$, et al. Transarterial chemoembolization (TACE) combined with sorafenib versus TACE for hepatocellular carcinoma with portal vein tumor thrombus: a systematic review and meta-analysis. Oncotarget. 2017;8(17): 29416-27.

64 Qiu Z, Shen L, Chen S, Qi H, Cao F, Xie L, et al. Efficacy of apatinib in transcatheter arterial chemoembolization (TACE) refractory intermediate and advanced-stage hepatocellular carcinoma: a propensity score matching analysis. Cancer Manag Res. 2019;11:9321-30

65 Choi GH, Shim JH, Kim MJ, Ryu MH, Ryoo BY, Kang YK, et al. Sorafenib alone versus sorafenib combined with transarterial chemoembolization for advanced-stage hepatocellular carcinoma: results of propensity score analyses. Radiology. 2013;269(2):603-11.

66 Cheng S, Chen M, Cai J, Sun J, Guo R, Bi X, et al. Chinese expert consensus on multidisciplinary diagnosis and treatment of hepatocellular carcinoma with portal vein tumor thrombus (2018 edition). Liver Cancer. 2020; 9(1):28-40 
67 Ikeda M, Shimizu S, Sato T, Morimoto M, Kojima $Y$, Inaba $Y$, et al. Sorafenib plus hepatic arterial infusion chemotherapy with cisplatin versus sorafenib for advanced hepatocellular carcinoma: randomized phase II trial. Ann Oncol. 2016;27(11):2090-6.

68 Kudo M, Ueshima K, Yokosuka O, Ogasawara S, Obi S, Izumi N, et al. Sorafenib plus low-dose cisplatin and fluorouracil hepatic arterial infusion chemotherapy versus sorafenib alone in patients with advanced hepatocellular carcinoma (SILIUS): a randomised, open label, phase 3 trial. Lancet Gastroenterol Hepatol. 2018;3(6):424-32.

69 Bekaii-Saab TS, Ou FS, Ahn DH, Boland PM, Ciombor KK, Heying EN, et al. Regorafenib dose-optimisation in patients with refractory metastatic colorectal cancer (ReDOS): a randomised, multicentre, open-label, phase 2 study. Lancet Oncol. 2019;20(8):1070-82.

70 Ye SL, Chen X, Yang J, Bie P, Zhang S, Liu F, et al. Safety and efficacy of sorafenib therapy in patients with hepatocellular carcinoma: final outcome from the Chinese patient subset of the GIDEON study. Oncotarget. 2016;7(6): 6639-48.

71 Ohki T, Sato K, Kondo M, Goto E, Sato T, Kondo $\mathrm{Y}$, et al. Impact of adverse events on the progression-free survival of patients with advanced hepatocellular carcinoma treated with lenvatinib: a multicenter retrospective study. Drugs Real World Outcomes. 2020; 7(2):141-9.

72 Shimose S, Iwamoto H, Niizeki T, Shirono T, Noda Y, Kamachi N, et al. Clinical significance of adverse events for patients with unresectable hepatocellular carcinoma treated with lenvatinib: a multicenter retrospective study. Cancers. 2020;12(7):1867.

73 Takahashi A, Moriguchi M, Seko Y, Ishikawa $\mathrm{H}$, Yo $\mathrm{T}$, Kimura $\mathrm{H}$, et al. Impact of relative dose intensity of early-phase lenvatinib treatment on therapeutic response in hepatocellular carcinoma. Anticancer Res. 2019;39(9): 5149-56.

74 Kirino S, Tsuchiya K, Kurosaki M, Kaneko S, Inada $\mathrm{K}$, Yamashita $\mathrm{K}$, et al. Relative dose intensity over the first four weeks of lenvatinib therapy is a factor of favorable response and overall survival in patients with unresectable hepatocellular carcinoma. PLoS One. 2020; 15(4):e0231828.

75 Ono A, Aikata H, Yamauchi M, Kodama K, Ohishi W, Kishi T, et al. Circulating cytokines and angiogenic factors based signature associated with the relative dose intensity during treatment in patients with advanced hepatocellular carcinoma receiving lenvatinib. Ther Adv Med Oncol. 2020;12:1758835920922051.

76 Kudo T, Kato T, Kagawa Y, Murata K, Ohta $\mathrm{H}$, Noura S, et al. Phase II dose titration study of regorafenib for patients with unresectable metastatic colorectal cancer who are progressed after standard chemotherapy. Ann Oncol. 2017;28:v208.
77 Argiles G, Margalef NM, Valladares-Ayerbes M, de Prado JV, Grávalos C, Alfonso PG, et al. Results of REARRANGE trial: a randomized phase 2 study comparing different dosing approaches for regorafenib (REG) during the first cycle of treatment in patients (pts) with metastatic colorectal cancer (mCRC). Ann Oncol. 2019;30:iv135.

78 Kim K, Jha R, Prins PA, Wang H, Chacha M, Hartley ML, et al. Regorafenib in advanced hepatocellular carcinoma (HCC): considerations for treatment. Cancer Chemother Pharmacol. 2017;80(5):945-54.

79 Yoo C, Park JW, Kim YJ, Kim DY, Yu SJ, Lim TS, et al. Multicenter retrospective analysis of the safety and efficacy of regorafenib after progression on sorafenib in Korean patients with hepatocellular carcinoma. Invest New Drugs. 2019;37(3):567-72.

80 Wang W, Tsuchiya K, Kurosaki M, Yasui Y, Inada K, Kirino S, et al. Sorafenib-regorafenib sequential therapy in Japanese patients with unresectable hepatocellular carcinoma-relative dose intensity and post-regorafenib therapies in real world practice. Cancers. 2019; 11(10): 1517.

81 Lim HY, Merle P, Finn RS, Frenette C, Masi $\mathrm{G}$, Ikeda M, et al. Regorafenib in patients with unresectable hepatocellular carcinoma (uHCC) in routine clinical practice: Interim analysis of the prospective, observational REFINE trial. J Clin Oncol. 2020;38(4_Suppl): 542.

82 Du R, Wang X, Ma L, Larcher LM, Tang H, Zhou $\mathrm{H}$, et al. Adverse reactions of targeted therapy in cancer patients: a retrospective study of hospital medical data in China. BMC Cancer. 2021;21(1):206.

83 Tian A, Zhou A, Bi X, Hu S, Jiang Z, Zhang $\mathrm{W}$, et al. Efficacy of topical compound danxiong granules for treatment of dermatologic toxicities induced by targeted anticancer therapy: a randomized, double-blind, placebocontrolled trial. Evid Based Complement Alternat Med. 2017;2017:3970601.

84 Lam W, Jiang Z, Guan F, Huang X, Hu R, Wang J, et al. PHY906(KD018), an adjuvant based on a 1800-year-old Chinese medicine, enhanced the anti-tumor activity of Sorafenib by changing the tumor microenvironment. Sci Rep. 2015;5:9384.

85 Barton DL, Liu H, Dakhil SR, Linquist B, Sloan JA, Nichols CR, et al. Wisconsin Ginseng (Panax quinquefolius) to improve cancer-related fatigue: a randomized, doubleblind trial, N07C2. J Natl Cancer Inst. 2013; 105(16):1230-8.

86 Zhang Y, Lin L, Li H, Hu Y, Tian L. Effects of acupuncture on cancer-related fatigue: a meta-analysis. Support Care Cancer. 2018;26(2): $415-25$.
87 Alsina A, Kudo M, Vogel A, Cheng AL, Tak WY, Ryoo BY, et al. Effects of subsequent systemic anticancer medication following firstline lenvatinib: a post hoc responder analysis from the phase 3 REFLECT Study in unresectable hepatocellular carcinoma. Liver Cancer. 2020;9(1):93-104.

88 Kudo M, Finn RS, Qin S, Han K-H, Ikeda K, Cheng A-L, et al. Analysis of survival and objective response (OR) in patients with hepatocellular carcinoma in a phase III study of lenvatinib (REFLECT). J Clin Oncol. 2019;37(4_ Suppl): 186 .

89 Vincenzi B, Di Maio M, Silletta M, D'Onofrio L, Spoto C, Piccirillo MC, et al. Prognostic relevance of objective response according to EASL criteria and mRECIST criteria in hepatocellular carcinoma patients treated with loco-regional therapies: a literature-based meta-analysis. PLoS One. 2015;10(7):e0133488.

90 Jung ES, Kim JH, Yoon EL, Lee HJ, Lee SJ, Suh SJ, et al. Comparison of the methods for tumor response assessment in patients with hepatocellular carcinoma undergoing transarterial chemoembolization. J Hepatol. 2013; 58(6):1181-7.

91 Wang P, Tan G, Zhu M, Li W, Zhai B, Sun X. Hand-foot skin reaction is a beneficial indicator of sorafenib therapy for patients with hepatocellular carcinoma: a systemic review and meta-analysis. Expert Rev Gastroenterol Hepatol. 2018;12(1):1-8.

92 Bruix J, Cheng A-L, Meinhardt G, Nakajima K, De Sanctis Y, Llovet J. Prognostic factors and predictors of sorafenib benefit in patients with hepatocellular carcinoma: analysis of two phase III studies. J Hepatol. 2017;67(5): 999-1008.

93 Marisi G, Cucchetti A, Ulivi P, Canale M, Cabibbo G, Solaini L, et al. Ten years of sorafenib in hepatocellular carcinoma: are there any predictive and/or prognostic markers? World J Gastroenterol. 2018;24(36): 4152-63.

94 Chuma M, Uojima H, Numata K, Hidaka $H$, Toyoda H, Hiraoka A, et al. Early changes in circulating FGF19 and Ang-2 levels as possible predictive biomarkers of clinical response to lenvatinib therapy in hepatocellular carcinoma. Cancers. 2020;12(2):293.

95 Yi C, Chen L, Lin Z, Liu L, Shao W, Zhang R, et al. Lenvatinib targets FGF receptor 4 to enhance antitumor immune response of antiprogrammed cell death-1 in HCC. Hepatology. 2021;74(5):2544-60.

96 Farsad K, Nabavizadeh N, Kardosh A, Jou JH, Naugler WE, Kolbeck KJ. Combined locoregional and systemic therapy for advanced hepatocellular carcinoma: finally, the future is obscure. Ann Transl Med. 2020;8(24):1700.

97 Davis GL, Dempster J, Meler JD, Orr DW, Walberg MW, Brown B, et al. Hepatocellular carcinoma: management of an increasingly common problem. Proceedings. 2008;21(3): 266-80. 